
\title{
25 Research Soure \\ Detection of Deletion/Insertion Polymorphism Profiles from Single Human Hair Shafts
}

Jian Tie ( $\nabla$ tetsu.ken@nihon-u.ac.jp )

Nihon University School of Medicine https://orcid.org/0000-0002-9797-8421

\section{Seisaku Uchigasaki}

Nihon University School of Medicine

\section{Eiji Isobe}

Nihon University School of Medicine

\section{Etsuko Iwakami}

Nihon University School of Medicine

\section{Takahisa Okuda}

Nihon University School of Medicine

\section{Research Article}

Keywords: Hair shaft, Low copy number, Genotype profile, Deletion/insertion polymorphisms, individual identification

Posted Date: June 22nd, 2021

DOl: https://doi.org/10.21203/rs.3.rs-619557/v1

License: (c) (i) This work is licensed under a Creative Commons Attribution 4.0 International License. Read Full License

Version of Record: A version of this preprint was published at Molecular Biology Reports on November 5th, 2021. See the published version at https://doi.org/10.1007/s11033-021-06921-w. 


\section{Abstract}

Background: Hair is a frequently encountered biological evidence in personal identification. The amount of nuclear DNA that can be extracted from a single strand of rootless hair is most limited, making the detection of short tandem repeat (STR) polymorphisms difficult. To overcome these limitations, deletion/insertion polymorphisms (DIP) as a new type of genetic marker have shown their benefits in detecting low-copy-number DNA. The Investigator DIPplex kit contains 30 biallelic autosomal DIP and amelogenin. The analysis of DIPs combines the advantages of both STR and single nucleotide polymorphism analyses. Thus, this study aimed to detect the DIP distribution of individual hair shafts from individuals.

Methods and Results: DNA was extracted from the shaft of fresh, aged, and shed hair. After DNA was evaluated, the DIP profiles were detected by capillary electrophoresis. The results indicated that the amount of DNA extracted from hair roots was much higher than that from the hair shafts in the same individual for all samples. The degradation index values of DNA from the aged hair shafts were highest. It is classified to be "mildly degraded." Compared with their hair roots, the full DIP profiles were detected for fresh hair, 70\% for aged hair, and 92\% for shed hair. Contrarily, except for fresh hair shafts, only three STR loci of the aged and shed strands of hair could be amplified using AmpFISTR MiniFiler PCR Amplification Kit.

Conclusions: These results indicate that the detection of DIP profile is an effective tool for personal identification from hair shafts, including aged hair.

\section{Introduction}

Hair is one of the common sources of DNA for personal identification such as that in forensic medicine. Human hair forms in the hair follicle, with 70-50 telogen strands of hair usually being lost per day [1]. Human hair comprises two parts: the hair root and the hair shaft. Fresh hair roots contain quite a number of somatic cells in which there is nuclear DNA (nuDNA). It can be used for personal identification via short tandem repeat (STR) polymorphism analysis [2-4]. Meanwhile, the hair shaft has only a small amount of scattered nuDNA, which is rarely sufficient for STR examination. In forensic medicine, most of the hair samples obtained from the scene for personal identification are naturally shed hair and typically do not contain hair roots. Even if there is a small number of somatic cells attached to the root of the hair, the number of somatic cells will decrease over time, making STR identification difficult.

The quality and quantity of recovered DNA and the presence of amplification inhibitors are often the key factors affecting DNA-based identification. For better detection and differentiation of these degraded DNAs, several commercial DNA kits have recently been developed on the basis of multiplex genetic markers; such kits include the AmpFISTR MiniFiler PCR Amplification Kit, the InnoTyper $21 \mathrm{Kit}$, and the Investigator DIPplex Kit. These markers reflect shorter nuDNA nucleotide sequences than those of commonly used STRs. These kits can amplify not only degraded DNA but also XY chromosome markers 
simultaneously. They have also been reported as powerful tools for individual identification based on degraded DNA [5-7].

Analysis of biallelic deletion/insertion polymorphisms (DIPs) combines the advantages of both STR and single nucleotide polymorphism analyses. DIPs are particularly useful in anthropological or population genetic studies. Additionally, DIP analysis confers further benefits, such as the elimination of stutter peaks, which enable mixed stain analysis [8]. The Investigator DIPplex Kit was developed for multiplex amplification of 30 biallelic DIPs plus amelogenin; these markers can easily be differentiated using capillary electrophoresis [9]. Indels are considered to be promising markers in forensic work. Thus, in the present study, we utilized the Investigator DIPplex Kit to examine the hair shafts of Japanese donors and compare the results of fresh and aged samples to evaluate the applicability of DIP analysis for personal identification on the basis of hair shaft DNA.

\section{Materials And Methods}

\section{Hair samples}

Plucked and naturally shed strands of hair from the scalp were collected from Japanese volunteers who were not related to each other. Fifty plucked fresh samples and naturally shed hair samples were temporarily stored at $-80^{\circ} \mathrm{C}$ until use. Additionally, a total of 21 plucked strands of hair were stored at room temperature for approximately 8 years. To know the difference in the amount of DNA with a single hair shaft, $2 \mathrm{~cm}$ and $5 \mathrm{~cm}$ lengths of different fresh hair shafts were cut for DNA extraction.

Approximately $1.5 \mathrm{~cm}$ of the single hair shaft was cut to obtain the hair root sample and then cut again at a site of approximately $2 \mathrm{~cm}$ or $5 \mathrm{~cm}$ from the previously cut portion to obtain a hair shaft sample. All hair samples were examined under a microscope to confirm the presence of hair roots. As control samples of DNA for comparison with the DIP profile of the hairs, we collected blood samples from the hair donors by venipuncture. Table 1 summarizes the collection and storage status of hair samples.

\section{DNA extraction and quantification}

Each DNA of hair roots and shafts was extracted using the QIAamp DNA Investigator Kit (Qiagen Inc., Valencia, CA, USA), following the manufacturer's instructions [10]. Before the DNA extraction, each hair shaft fragment was washed with $100 \%$ ethanol (the hair roots were not washed with ethanol), and the DNA was eluted with $50 \mu \mathrm{L}$ of distilled water. DNA extraction of the blood was performed using the QIAamp DNA Blood Investigator Kit (QIAGEN Inc.).

The quality and quantity of recovered DNA were assessed by the Quantifiler ${ }^{\circledR}$ Trio DNA Quantification Kit (Thermo Fisher Scientific, Oyster Point, CA, USA) according to the manufacturer's instructions [11]. The primary quantification targets (small autosomal and $Y$ ) consist of relatively short amplicon to improve the detection of degraded DNA samples. Also, the kits each contain a large autosomal target with a longer amplicon to aid in determining if a DNA sample is degraded. The human DNA that is included in the kit used to generate the DNA quantification standard dilution series comprised pooled human male 
genomic DNA. Quantitative PCR was performed using the QuantStudio ${ }^{\mathrm{TM}} 5$ Real-Time PCR system (Thermo Fisher Scientific), and the degradation index (DI) was calculated according to the manufacturer's protocol and software.

\section{Amplification and analysis}

Thirty biallelic autosomal indels and amelogenin were amplified using the Investigator ® DIPplex Kit (Qiagen), per the manufacturer's protocols, using the ProFlex ${ }^{\text {TM }}$ PCR system thermal cycler (Applied Biosystems). The kit was developed for multiplex amplification of 30 biallelic DIPs plus Amelogenin. PCR reactions were conducted in volumes of $25 \mu \mathrm{L}$, containing $5 \mu \mathrm{L}$ of reaction Mix A, $5 \mu \mathrm{L}$ of Primer Mix, 0.6 $\mu \mathrm{L}$ of Multi Taq2 DNA Polymerase, and $14.4 \mu \mathrm{L}$ of template DNA (Most 5-cm-long fresh hair samples were collected with more than $5 \mathrm{pg} / \mu \mathrm{L}$ DNA). The PCR cycling protocol was based on the following condition: $94^{\circ} \mathrm{C}$ for $4 \mathrm{~min} ; 32$ cycles of $94^{\circ} \mathrm{C}$ for $30 \mathrm{~s}, 61^{\circ} \mathrm{C}$ for $120 \mathrm{~s}$, and $72^{\circ} \mathrm{C}$ for $75 \mathrm{~s}$; amplification was terminated via a final step at $68^{\circ} \mathrm{C}$ for $60 \mathrm{~min}$. PCR products denatured using Hi-Di formamide were analyzed using the ABI Prism 310 capillary electrophoresis system (Applied Biosystems), followed by DNA sequence data analysis using GeneMapperID v3.2 (Life Technologies).

Generate standard curves were created using the Quantifiler THP DNA standard genomic DNA (100 ng / $\mu \mathrm{L}$ ) included in the kit, and the software was set to recognize only above 50 relative fluorescent units RFU as a peak of DIP.

STR markers were amplified from hair samples using the AmpFISTR MiniFiler PCR Amplification Kit (Applied Biosystems), PCR amplification, and capillary electrophoresis were performed following the manufacturer's protocols. The PCR products were analyzed using the ABI Prism 310 capillary electrophoresis system.

\section{Results}

To assess the quantity and quality of hair DNA from Japanese donors, $5 \mathrm{~cm}$ of long fresh, aged, and shed hair and a single root were tested. Additionally, another $2 \mathrm{~cm}$ of fresh hair shaft was used to compare the amount of DNA. Figure 1A presents the average concentration of DNA extracted from different hair samples. As shown in the data, the amount of DNA obtained from the hair shaft with a length of $2 \mathrm{~cm}$ was much lower than that from the fresh hair shaft with a length of $5 \mathrm{~cm}$; hence, it was not used for the examination of the aged strands of hair and the shed strands of hair. Whether it was fresh, aged, or naturally shed hair, the amount of DNA extracted from hair roots was much higher than that from the hair shafts. Particularly, the DNA content of fresh hair roots was more than 2,000 times higher than that of the hair shafts. For aged samples and naturally shed hair, the DNA content of the hair roots was more than 200 times higher than that of the hair shafts. Additionally, considerable variations were observed in the amount of DNA obtained between different individuals, especially regarding DNA from the fresh hair roots, in which the maximum amount was approximately seven times the minimum amount. Given that the amount of DNA extracted from the hair shafts was too small, we attempted to use more distilled 
water to recover DNA from the filter membrane when extracting DNA. Before PCR amplification, we kept the lid of the DNA test tube open and placed the tube in a refrigerator $\left(4^{\circ} \mathrm{C}\right)$ to let the water evaporate, thereby further concentrating the DNA, and then determine the respective DNA concentrations.

The Quantifiler Trio DNA Quantification Kit was used to evaluate the DI of all hair samples. (Fig. 1B). The average DI values of DNA from fresh hair were below 1 for both the roots and shafts. Although the DI values of DNA from the aged hair shafts were highest in all samples, they did not exceed a DI value of 4 , and the DNA from these samples could be categorized as "mildly degraded." Hence, the level of degradation of DNA from aged shafts should not have too much influence on the detection of the DIP according to the manufacturer's protocols. Additionally, the range in DI values for aged hair and naturally shed hair was relatively high, especially for hair shaft samples; this could be due to the differences between individuals [12].

Although the root size of aged or shed hair was found to vary from individual to individual, both the amplified DIP profiles reached approximately $90 \%$. Although the DI values of DNA from aged hair shafts were highest among all samples, it is also "mildly degraded" in the DI classification. The results of DIP detection of hair roots were showed complete for fresh strands of hair, $70 \%$ for aged strands of hair, and $92 \%$ for shed hair (Fig. 2). This means that although the DIP detection rate of aged hair roots is lower than that of fresh and shed hair roots, most of the loci may still be amplified by the profiler. For fresh hair, the complete loci of DIP were confirmed in the 5-cm-long hair shafts, whereas only $30 \%$ DIP loci were detected in the 2-cm-long hair shafts. Thus, to amplify the DIP profile from a single hair shaft, a certain length of the hair shaft is required. As shown in Fig. 2, complete DIP profiles detected in each blood samples, inconsistent genotypes could not be confirmed by comparing the DIP profiles from the blood, hair roots and hair shafts of all hair donors.

The number of detected DIP alleles and their relationship with DI were evaluated from all hair samples. The numbers of the DIP alleles in the hair shafts were amplified between 14 and 26 (mean = 19.3, S.D.= 3.1) for the aged hairs, 23 and 30 (mean $=25.4$, S.D. $=2.3$ ) for the shed hairs. As the DI value increases, fewer DIP alleles are amplified, especially in the aged hair (Fig. 3). The DI value was highest for aged hair, followed by shed hair shaft. For detection of DIP alleles, only an average of 19 was amplified on the aged hair shaft, but an average of 25 on the shed hair shaft. From this study, it was suggested that the DNA of hair shafts were degradation after stored for a long time, and that the number of DIP alleles was detected but decreased.

When testing different portions of the same sample (root $v s$. shaft), it was observed that some DIP alleles amplified from the hair root DNA were not amplified from the corresponding hair shaft DNA. For example, as Fig. 4 presents, a total of 15 peaks were amplified from an aged hair root sample excluding the amelogenin locus (Fig. 4A) in the blue channel loci; however, only 12 peaks were detected in the DNA from the hair shaft; thus, alleles D131+, D56-, and D56 + were not detected in the hair shaft DNA (Fig. 4B). In the same way, another channel presents 12 peaks amplified from a naturally shed hair root sample (Fig. 4C); however, only 10 alleles were amplified from the hair shaft, with the alleles D39 - and 
D39 + missing (Fig. 4D). Hence, the allele dropouts in some aged and shed hair shafts are considered to be the main causes of the decrease in the DIP locus in this study.

The MiniSTR multiplex PCR for the analysis of degraded DNA has been described previously $[13,14]$. The AmpFISTR MiniFiler PCR Amplification Kit amplifies eight autosomal STR loci and amelogenin in a single PCR reaction; the loci span a range of 70-280 nucleotides. Regarding DNA from fresh hair root samples, complete MiniFiler STR profiles could be amplified; however, no more than four STR loci could be detected in other types of hair.

\section{Discussion}

Analysis of DIP profile is similar to STR. The peak height is related to the amount of the amplified product. STR target amplicons usually range from 100 to 400 base pairs (bp) in length; however, the amplicon size of the hair shaft is usually less than $100 \mathrm{bp}$, which is because of the degradation of organelles and nucleic acids during the keratinization process [15]. The effect of DIP profile amplification due to the increase in DI value was shown. Approximately $37 \%$ of alleles were not detected, especially in aged hair shafts, but the complete DIP profiles were found in fresh hair shafts, and more than $83 \%$ of alleles were accurately amplified in shed hair shafts. Many of the evidence materials have no hair roots, and the results of this study showed that the number of DIP alleles detected in aged hair shafts decreased, but a certain number of alleles could be confirmed. STR is widely used for personal identification, but it is quite difficult for hair shafts without hair roots. In this study, DIP obtained genetic information from the hair shaft more than MiniFiler STR, and it was found that it is a significant method even for aged hair shafts.

Regarding the sensitivity of DIP detection, the complete profiles were reported to be as low as $8 \mathrm{pg}$ of template DNA [16]. We used the positive control DNA9948, for which, at least an approximately $10 \mathrm{pg}$ template is required. However, for the amplification of DIP loci from aged hair shaft samples, more than $15 \mathrm{pg}$ of template DNA proved necessary, perhaps because of the quantity and quality of DNA.

The amplification of LCN samples often results in at least two artifacts: One is an allele drop-in. This is the case when alleles are accidentally added because of contamination. The other is the allele dropout, which mostly happens because of insufficient DNA, resulting in some alleles not being able to be amplified. However, allele drop-ins are usually not reproducible, and the probability of detecting the same drop-in allele is less than $1 \%$ when two individual PCR reactions are performed $[17,18]$. Thus, in this study, the DIP profiler was run twice to confirm reproducibility. These results show that DIP analysis is an effective personal identification tool, when using not only DNAs extracted from fresh hair shafts but also those from the naturally shed strands of hair and the aged strands of hair.

\section{Conclusions}


Because of the limited amount of nuDNA in a single hair shaft, amplifying multiple STR markers for personal identification is difficult. In this study, fresh hair, aged hair preserved at room temperature, and naturally shed hair were compared in terms of the detection of DIP and STR. The DNA content in the hair shaft was very little, and the DNA quality also declined following long-term storage; however, more genetic information could be obtained by DIP profilers than by STR profilers. No difference was observed in comparing the DIP profiles of the roots and hair shafts and in the two times reproducibility tests for each sample. Thus, when only the hair shafts are available and used as evidence of DNA, DIP may be a new method of choice.

\section{Declarations}

\section{Funding}

This work was supported by Japan Society for the Promotion of Science KAKENHI, Great-in-Aid for Scientific Research (C) number 21K10533.

\section{Conflict of interest}

The authors declare no conflicts of interest.

\section{Availability of data and material}

Data produced during this study are available from the corresponding author upon reasonable request. No specific materials were produced during this study.

\section{Ethics approval}

This study was approved by the ethical committee of the Nihon University in accordance with ethical guidelines. (Decision No: 239-0). All procedures performed in studies involving human participants were in accordance with the ethical standards of this Ethics Committee and with the 1964 Helsinki declaration and its later amendments or comparable ethical standards. Informed consent was obtained from all participants included in the study.

\section{Consent to participate}

All the authors listed have approved the manuscript that is enclosed.

\section{Consent for publication}

The manuscript is approved by all authors for publication.

\section{Acknowledgements}

We would like to extend our thanks to Dr. Hirofumi Tsutsumi for his comments on the DNA evaluation of the samples. 


\section{References}

1. Müller K, Klein R, Miltner E et al (2007) Improved STR Typing of Telogen Hair Root and Hair Shaft DNA. Electrophoresis 28(16):2835-2842

2. Brandhagen DM, Loreille O, Irwin JA (2018) Fragmented Nuclear DNA Is the Predominant Genetic Material in Human Hair Shafts. Genes (Basel) 9(12):640-660

3. Ottens R, Taylor D, Abarno D et al (2013) Successful direct amplification of nuclear markers from a single hair follicle. Forensic Sci. Med. Pathol. 2013, 9: 238-243

4. Parson w, Huber G, Moreno L et al (2015) Massively parallel sequencing of complete mitochondrial genomes from hair shaft samples. Forensic Sci Int Genet 15:8-15

5. Luce C, Montpetit S, Gangitano D et al (2009) Validation of the AmpFISTR MiniFiler PCR Amplification Kit for Use in Forensic casework. J Forensic Sci 54(5):1046-1054

6. Grisedale KS, Murphy GM, Brown H et al (2018) Successful nuclear DNA profiling of rootless hair shafts: a novel approach. Int J Legal Med 132(1):107-115

7. Oldoni E, Podini D (2019) Forensic Molecular Biomarkers for Mixture Analysis. Forensic Sci Int Genet 41:107-119

8. Investigator DIPplex Handbook. November (2014)

9. Fondevila M, Phillips C, Santos C et al (2012) Forensic Performance of Two Insertion-Deletion Marker Assays. Int J Legal Med 126(5):725-737

10. QIAamp® DNA Investigator Handbook (2020)

11. Quantifiler TMHP, Trio DNA Quantification Kits, user guide (2018)

12. Bengtsson CE, Olsen ME, Brandt L et al (2012) DNA From Keratinous Tissue. Part I: Hair and Nail. Ann Anat 194(1):17-25

13. Mulero JJ, Chang CW, Lagacé RE et al (2008) Development and validation of the AmpFISTR MiniFiler PCR Amplification Kit: a MiniSTR multiplex for the analysis of degraded and/or PCR inhibited DNA. J Forensic Sci 53(4):838-852

14. Horsman-Hall KM, Orihuela Y, Karczynski SL et al (2009) Development of STR profiles from firearms and fired cartridge cases. Forensic Sci Int Genet 3(4):242-250

15. Brandhagen MD, Loreille O, Irwin J (2018) Fragmented nuclear DNA is the predominant genetic material in human hair shafts. Genes 9(640):1-20

16. Klein R, Neumann C, Roy R (2015) Detection of insertion/deletion polymorphisme from challenged samples using Investigator DIPplex Kit. Forensic Sci Int Genet 16:29-37

17. Taberlet P, Griffin S, Goossens B B et al (1996) Reliable genotyping of samples with very low DNA quantities using PCR. Nucleic Acids Res 24(16):3189-3194

18. Whitaker JP, Cotton EA, Gill P (2001) A comparison of the characteristics of profiles produced with the AmpFISTR SGM Plus multiplex system for both standard and low copy number STR DNA analysis. Forensic Sci Int 123(2-3):215-223 


\section{Tables}

Due to technical limitations, table 1 is only available as a download in the Supplemental Files section.

\section{Figures}

A

B
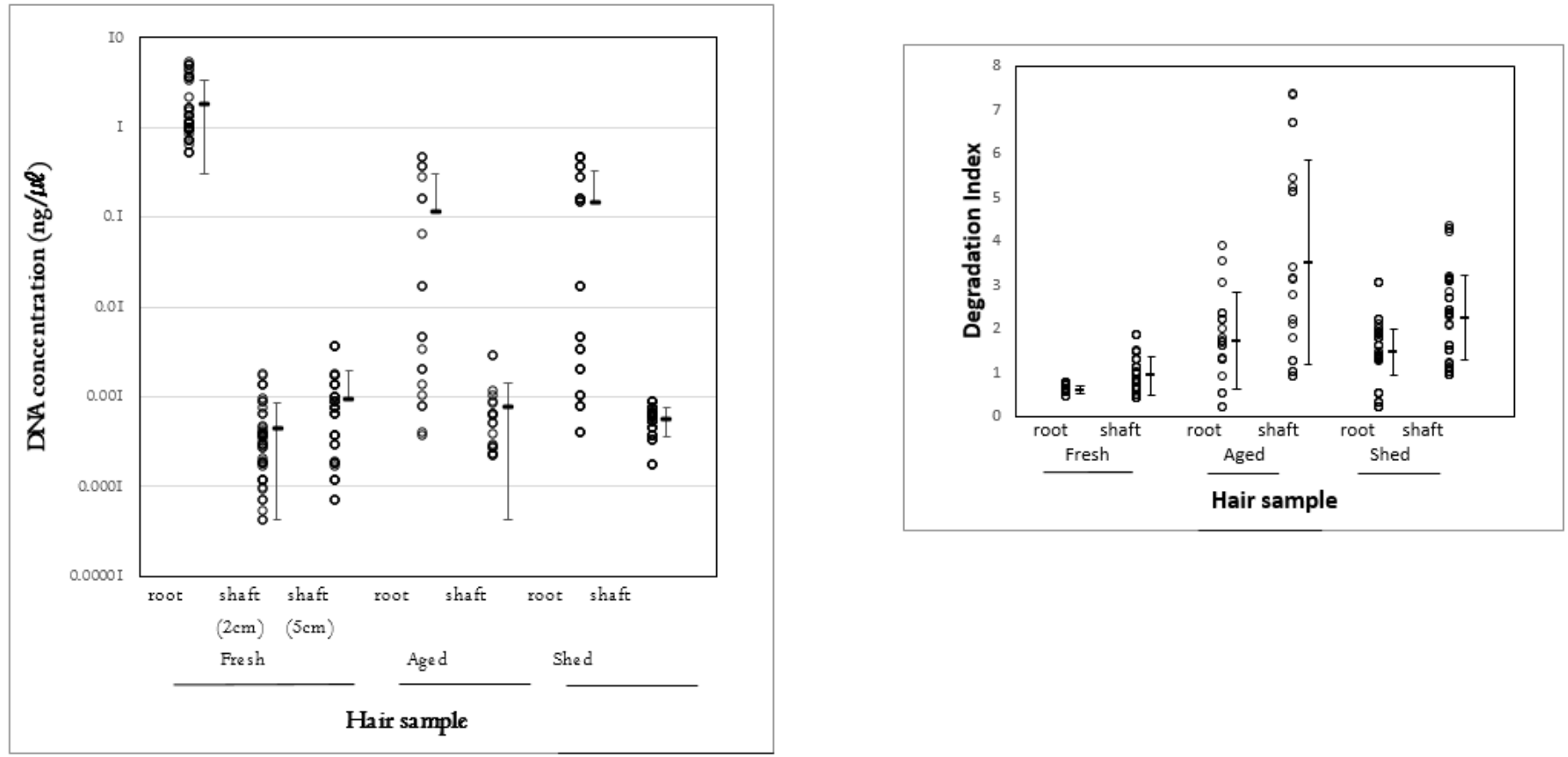

\section{Figure 1}

A: Average DNA concentration of a single hair root and its hair shaft in freshly plucked, aged and naturally shed hair samples. The amount of DNA obtained from the hair roots were much higher than the hair shafts. The amount of DNA obtained from the $2 \mathrm{~cm}$ long hair shaft was less than the $5 \mathrm{~cm}$ long aged hair shafts and shed hair shaft. B: Average DI of each hair DNA. It was less than 2 for hair roots and fresh hair shafts and more than 2 for aged hair shafts and shed hair shafts. 


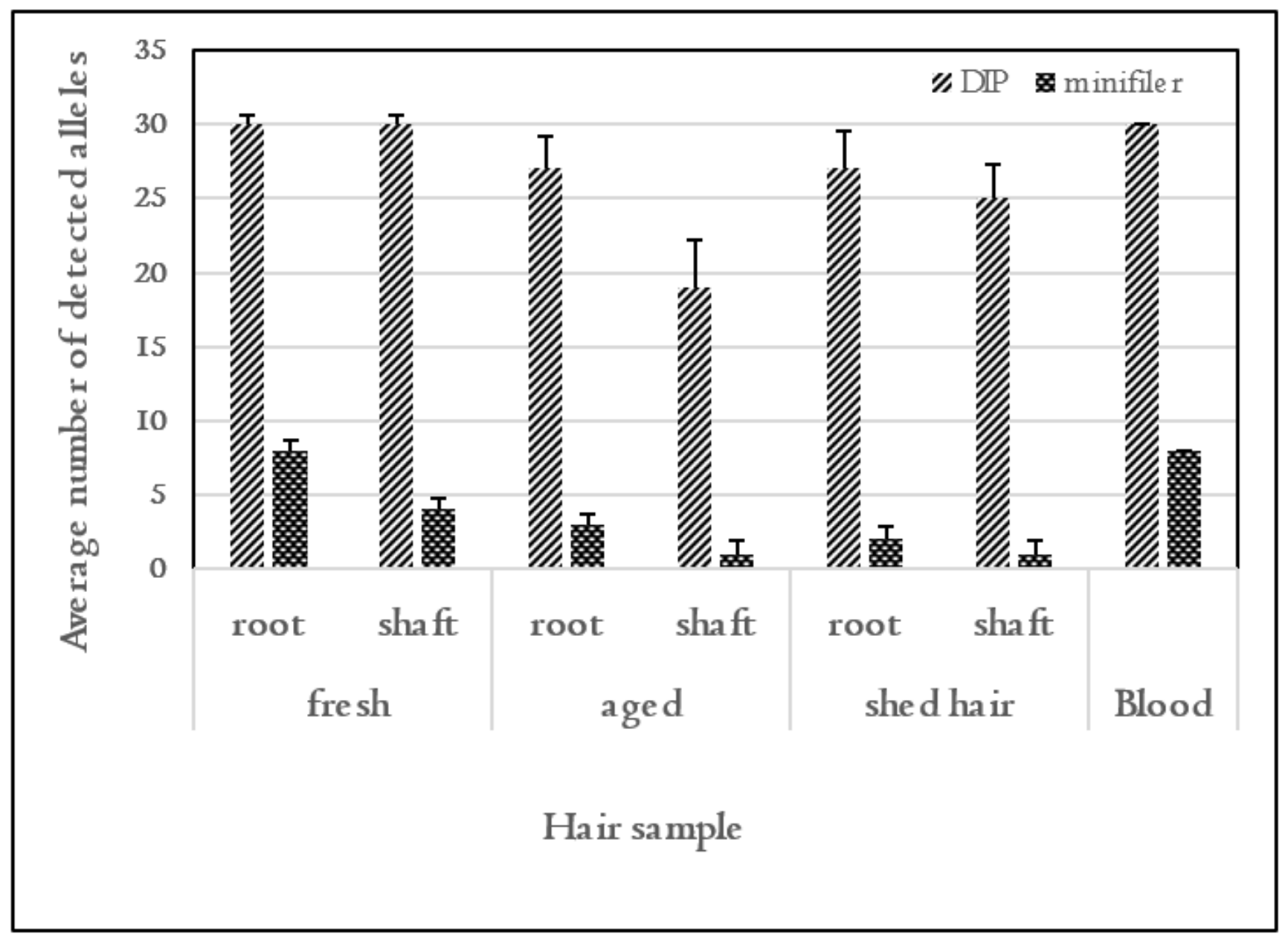

Figure 2

Detected average numbers of DIP loci and MiniFiler loci from a single hair sample. The detected locus was more in DIP than in MiniFiler in all samples. 


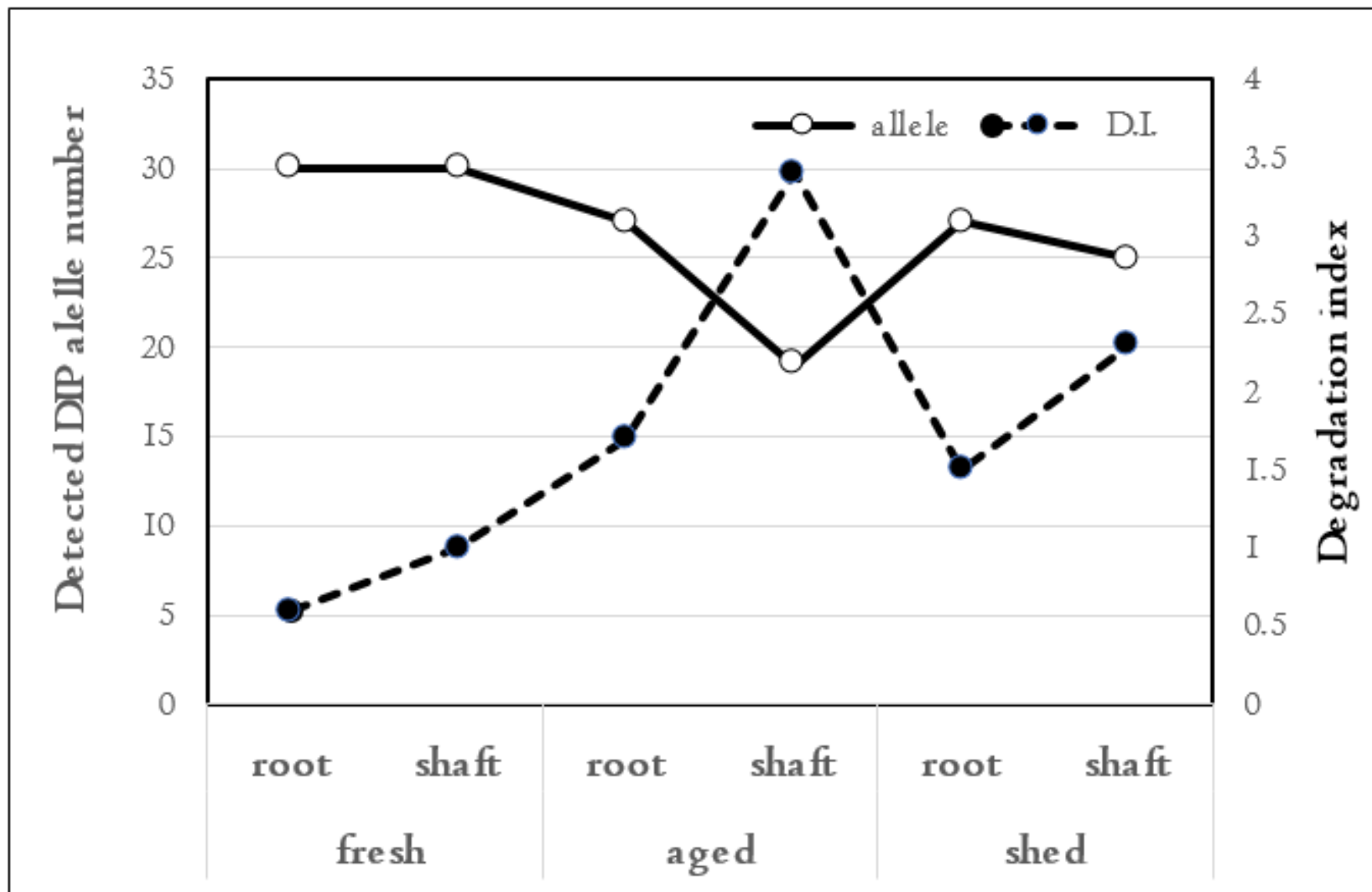

Hair sample

Figure 3

Analysis of the relationship between changes in DI and amplified alleles. In hair shaft samples, when DI values increased that amplified the number of DIP alleles reduced. 
A

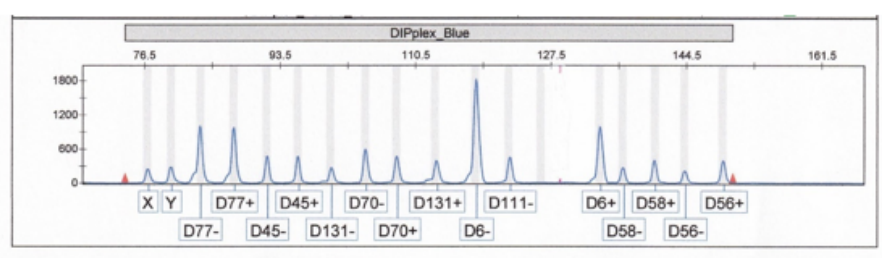

B

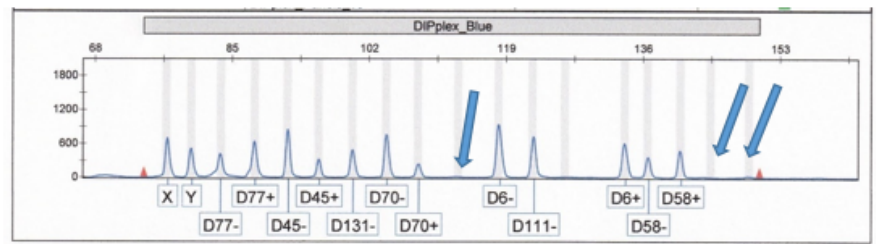

$\mathbf{C}$

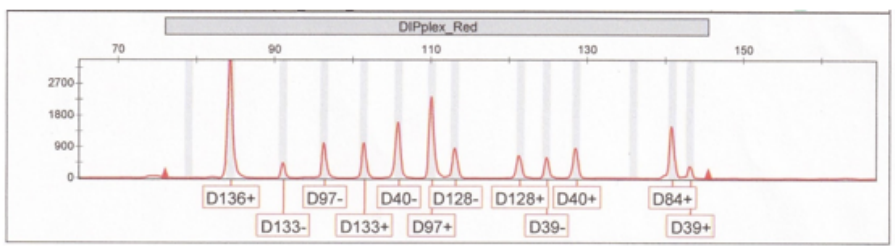

D

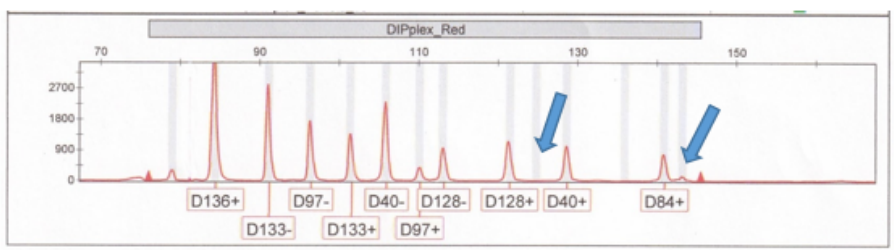

Figure 4

$A$ \& B: Electropherograms of a single aged hair root $(A)$ and its hair shaft $(B)$ amplified with the Investigator ${ }^{\circledR}$ DIPplex Kit for Blue channel loci. The alleles of D131 +, D56- and D56 + amplified in the root of aged hair did not appear in the hair shaft. C \& D: Electropherograms of a single shed hair root (C) and its shed shaft (D) amplified with the Investigator ${ }^{\circledR}$ DIPplex Kit for Red channel loci. The alleles of D39- and D39 + amplified in the root of shed hair did not appear in the hair shaft.

\section{Supplementary Files}

This is a list of supplementary files associated with this preprint. Click to download.

- Table1.xlsx 